\title{
Integral Sliding Mode Control for Trajectory Tracking of Wheeled Mobile Robot in Presence of Uncertainties
}

\author{
Aicha Bessas, ${ }^{1}$ Atallah Benalia, ${ }^{1}$ and Farès Boudjema ${ }^{2}$ \\ ${ }^{1}$ LACoSERE Laboratory, Amar Telidji University, Laghouat, BP 37G Route de Ghardaïa, 03000 Laghouat, Algeria \\ ${ }^{2}$ LCP Laboratory, Ecole Nationale Polytechnique, 10 avenue Hassan Badi, BP 182, El-Harrach, Algiers, Algeria
}

Correspondence should be addressed to Aicha Bessas; ai.bessas@lagh-univ.dz

Received 9 November 2015; Revised 25 April 2016; Accepted 28 April 2016

Academic Editor: Yongji Wang

Copyright (C) 2016 Aicha Bessas et al. This is an open access article distributed under the Creative Commons Attribution License, which permits unrestricted use, distribution, and reproduction in any medium, provided the original work is properly cited.

\begin{abstract}
Wheeled mobile robots present a typical case of complex systems with nonholonomic constraints. In the past few years, the dominance of these systems has been a very active research field. In this paper, a new method based on an integral sliding mode control for the trajectory tracking of wheeled mobile robots is proposed. The controller is designed to solve the reaching phase problem with the elimination of matched disturbances and minimize the unmatched one. We distinguish two parts in the suggested controller: a high-level controller to stabilize the nominal system and a discontinuous controller to assess the trajectory tracking in the presence of disturbances. This controller is robust during the entire motion. The effectiveness of the proposed controller is demonstrated through simulation studies for the unicycle with matched and unmatched disturbances.
\end{abstract}

\section{Introduction}

Generally, wheeled mobile robots (WMRs) are the most widely used classes of mobile robots. This is due to their practical importance and theoretically interesting properties. These systems are a typical example of nonholonomic mechanisms where the constraints imposed on the motions are not integrable resulting from the assumption that there is no slipping of the wheels. The main consequence of a nonholonomic constraint for the WMRs is that not each path of the admissible configuration space corresponds to a feasible trajectory for the robot. In the literature of the wheeled mobile robot control, there are two fundamental problems: posture stabilization and trajectory/path tracking. The aim of posture stabilization is to stabilize the robot to a desired point [1], while the trajectory tracking is to enforce the robot to follow a reference trajectory [2]. For WMRs, it is difficult to control such system by continuous time-invariant controller. This is due to the uncontrollability of their linear approximation and to Brockett's necessary condition, which is not satisfied for this kind of system [3]. To overcome these difficulties, various control strategies have been investigated among them: homogeneous and timevarying feedback [4], sinusoidal and polynomial controls
[5], backstepping approaches [6, 7], and hybrid controls [8]. In the real implementation, it is desired to design an inherently robust control which provides fast convergence and good robustness properties with respect to the parameter variation and the disturbances. One of the robust techniques is the discontinuous control such as sliding mode control (SMC). There are a number of references on sliding mode control devoted to this type of discontinuous control, the best known one of which is by Utkin et al. [9]. More theoretical analyses and comparison study of performances for different SMC controllers are presented in [10]. The sliding mode control has many advantages, among them, its finite time convergence to a stable manifold and its insensitivity to the disturbances and model uncertainties satisfying the matching condition. However, it has some disadvantages such as the chattering phenomena, the reaching phase, and sensitivity to the unmatched perturbation. To enhance the robustness of the sliding mode control in the whole motion, it is interesting to eliminate the reaching phase and minimize the effect of the unmatched disturbances. This idea can be done by applying the integral sliding mode design concept proposed in $[9,11-$ 14]. The integral sliding mode control seeks to eliminate the reaching phase by enforcing sliding mode throughout 
the entire system response. The basic idea of this control (ISMC) is the inclusion of an integral term to the sliding manifold. This integral term enables the system to start on the sliding manifold at the initial condition, hence eliminating the reaching phase. From the integral sliding manifold, we define two controllers [9]: a continuous control and a discontinuous control. The continuous controller is a nonlinear continuous feedback designed to stabilize the nominal system and the discontinuous control is used to reject the matched disturbances and minimize the unmatched one.

The main objective of this work is the design of a robust controller for the trajectory tracking of the unicycle subject to state-dependent uncertainties (matched and unmatched). To attain this objective, we use an integral sliding mode based controller. This suggested controller combining nonlinear time-varying feedback with an integral sliding mode controller. An integral sliding mode controller is constructed by incorporating an integral term in the switching manifold.

The outline of this paper is as follows. In Section 2, the problem statement and the integral sliding mode controller design are presented for nonlinear uncertain system. Then, the kinematic model of the unicycle-type wheeled mobile robot is derived in Section 3. In Section 4 the design of integral sliding mode controller for tracking control of the unicycle is presented. Then, some simulation results are discussed in Section 5. Finally, Section 6 concludes this paper.

\section{Problem Statement}

Consider the following nonlinear uncertain system:

$$
\dot{q}_{e}=f\left(q_{e}, t\right)+g\left(q_{e}\right) u+P\left(q_{e}, t\right),
$$

where $q_{e} \in Q \subset \operatorname{IR}^{n}$ is the state of the system with initial condition $q_{e}\left(t_{0}\right)=q_{0}$ and $u \in \mathrm{IR}^{m}$ is the control variable. The function $f \in \mathrm{IR}^{n}$ is a known vector and the matrix $g \in \mathrm{IR}^{n \times m}$ is a known full rank state-dependent matrix. We suppose also that the origin is an equilibrium point of (1); that is, $f(0, t)=0 \forall t>0 . P\left(q_{e}, t\right) \in \mathrm{IR}^{n}$ is an unknown vector representing the modeling uncertainties and external disturbances. The following assumption is introduced.

Assumption 1. The uncertain vector $P\left(q_{e}, t\right)$ is bounded:

$$
\begin{aligned}
P\left(q_{e}, t\right) & \in \Phi, \\
\Phi & \triangleq\left\{v \in \mathrm{IR}^{n} \text { s.t. }\|v\|_{2} \leq D_{\text {sup }}\right\},
\end{aligned}
$$

where $D_{\text {sup }}$ is a known positive constant.

The uncertain vector $P\left(q_{e}, t\right)$ for system (1) can always be expressed by separating the matched disturbance $P_{M}\left(q_{e}, t\right)$ and the unmatched one $P_{U}\left(q_{e}, t\right)$, as follows:

$$
\begin{gathered}
P\left(q_{e}, t\right)=P_{M}\left(q_{e}, t\right)+P_{U}\left(q_{e}, t\right), \\
P_{M}\left(q_{e}, t\right)=g\left(q_{e}\right) g^{+}\left(q_{e}\right) P\left(q_{e}, t\right), \\
P_{U}\left(q_{e}, t\right)=g^{\perp}\left(q_{e}\right) g^{\perp+}\left(q_{e}\right) P\left(q_{e}, t\right),
\end{gathered}
$$

where $g^{\perp}\left(q_{e}\right) \in \operatorname{IR}^{n \times(n-m)}$ is a matrix with independent columns that span the null space of $g\left(q_{e}\right)$ : that is, $g^{\perp T}\left(q_{e}\right) g\left(q_{e}\right)=0_{(n-m) \times m}, \operatorname{Rank}\left(g^{\perp}\left(q_{e}\right)\right)=n-m$. Moreover, $g^{+}\left(q_{e}\right)$ is the left pseudoinverse of $g\left(q_{e}\right)$; that is, $g^{+}\left(q_{e}\right)=$ $\left(g^{T}\left(q_{e}\right) g\left(q_{e}\right)\right)^{-1} g^{T}\left(q_{e}\right)$, analogously for $g^{\perp+}\left(q_{e}\right)$. This separation principle relies on proposition 1 [15], which ensures that $I_{n}=g\left(q_{e}\right) g^{+}\left(q_{e}\right)+g^{\perp}\left(q_{e}\right) g^{\perp+}\left(q_{e}\right)$ for any full rank $g\left(q_{e}\right)$, being $I_{n} \in \mathrm{IR}^{n \times n}$ an identity matrix.

Our aim is to construct a robust feedback controller, which makes system (1) asymptotically stable. More precisely, for a given known stabilizing control for the nominal system of (1), we want to redesign another robust stabilizing feedback control of the perturbed system (1). We can realize that we want to robustify an existing feedback control of the nominal system. To attempt this objective, we will take into account the following assumption.

Assumption 2. The system nominal part of (1)

$$
\dot{q}_{e}=f\left(q_{e}, t\right)+g\left(q_{e}\right) u
$$

is globally asymptotically stabilizable via a nonlinear timevarying continuous control $u_{c}\left(q_{e}, t\right)$.

Since the control $u_{c}\left(q_{e}, t\right)$ is supposed to be not robust with respect to dynamic (1) and to enhance the robustness, we will add to it an integral sliding mode controller, which guarantees a good robustness during the entire motion of the states of the obtained closed-loop system.

2.1. Integral Sliding Mode Controller Design. The enhancement of the robustness of the feedback control $u_{c}\left(q_{e}, t\right)$ is done by using the integral sliding mode controller to reject the perturbations while eliminating the reaching phase. The integral sliding mode algorithm is designed in two design steps $[12,13]$ as follows.

(1) The design of a suitable integral sliding manifold $s\left(q_{e}, t\right)$ satisfying the control objectives on the sliding mode.

(2) The design of corresponding control input $u$ constraining the system trajectories to evolve on the integral sliding surface from the initial time and make the feedback system insensitive to the disturbances.

The integral sliding function can be defined as

$$
s\left(q_{e}, t\right)=s_{0}\left(q_{e}, t\right)+z_{s}\left(q_{e}, t\right),
$$

where $s \in \mathrm{IR}^{m}, s_{0} \in \mathrm{IR}^{m}$ is designed as the linear or nonlinear function of the system states, and $z_{s} \in \mathrm{IR}^{m}$ is an unknown integral function of the state to be determined such that the reaching phase is eliminated. The integral sliding manifold is given by $s\left(q_{e}, t\right)=0$.

Differentiating $s$ in (6) yields

$$
\begin{aligned}
& \dot{s}\left(q_{e}, t\right)=H\left(q_{e}\right)\left[f\left(q_{e}, t\right)\right. \\
& \left.\quad+g\left(q_{e}\right)\left(u+g^{+}\left(q_{e}\right) P\left(q_{e}, t\right)\right)+P_{U}\left(q_{e}, t\right)\right]+\dot{z}_{s}
\end{aligned}
$$

where $H\left(q_{e}\right)=\partial s_{0}\left(q_{e}\right) / \partial q_{e} \in \mathrm{IR}^{m \times n}$. 
In absence of perturbation, the invariance conditions of the integral sliding manifold with the nonlinear time varying continuous control $u_{c}\left(q_{e}, t\right)$ are given by

$$
\begin{aligned}
s\left(q_{e}, t\right) & =0 \quad \forall t>0, \\
\dot{s}\left(q_{e}, t\right) & =0 \quad \forall t>0 \Longrightarrow \\
\dot{z}_{s} & =-H\left(q_{e}\right)\left[f\left(q_{e}, t\right)+g\left(q_{e}\right) u_{c}\right] .
\end{aligned}
$$

To satisfy this invariance condition from the initial time, we obtain from the above equations the dynamics of the variable $z_{s}$ :

$$
\begin{aligned}
\dot{z}_{s} & =-H\left(q_{e}\right)\left[f\left(q_{e}, t\right)+g\left(q_{e}\right) u_{c}\right], \\
z_{s}(0) & =-s_{0}\left(q_{e}\left(t_{0}\right)\right) .
\end{aligned}
$$

According to (6), (9), we obtain

$$
\begin{aligned}
s= & s_{0}\left(q_{e}, t\right)-s_{0}\left(q_{e}\left(t_{0}\right)\right) \\
& -\int_{t_{0}}^{t} H\left(q_{e}\right)\left[f\left(q_{e}, \tau\right)+g\left(q_{e}\right) u_{c}\right] d \tau .
\end{aligned}
$$

Note that this integral sliding manifold is analogous to those proposed in [11]. We can see that the invariance condition is verified for this sliding surface with the control $u_{c}\left(q_{e}, t\right)$ if the disturbance does not appear. In order to guarantee the attractivity of the sliding manifold (10) and the robustness against the perturbation, we will add a discontinuous control part to the time varying continuous control $u_{c}\left(q_{e}, t\right)$. We put the robust feedback control in the following form:

$$
u\left(q_{e}, t\right)=u_{c}\left(q_{e}, t\right)+u_{\mathrm{disc}}\left(q_{e}, t\right),
$$

where $u_{c}\left(q_{e}, t\right)$ is the feedback stabilizing control of the nominal system (5) which guarantees the invariance of the integral sliding manifold. $u_{\text {disc }}\left(q_{e}, t\right)$ is a discontinuous control action designed to minimize the disturbance terms, forcing the system state on a suitably designed integral sliding manifold $s\left(q_{e}, t\right)=0$.

Assumption 3. $H\left(q_{e}\right)$ is such that

$$
\operatorname{Rank}\left(H\left(q_{e}\right) g\left(q_{e}\right)\right)=m \quad \forall q_{e} \in \mathrm{IR}^{n} .
$$

Take into account the reachability condition defined as follows [9]:

$$
\dot{S}=-M \cdot \operatorname{sign}(S) .
$$

The discontinuous control is

$$
u_{\mathrm{disc}}\left(q_{e}, t\right)=-M \frac{\left(H\left(q_{e}\right) g\left(q_{e}\right)\right)^{T} s\left(q_{e}, t\right)}{\left\|\left(H\left(q_{e}\right) g\left(q_{e}\right)\right)^{T} s\left(q_{e}, t\right)\right\|_{2}},
$$

where $M=\left[\begin{array}{cc}M_{1} & 0 \\ 0 & M_{2}\end{array}\right] \in \mathrm{IR}^{m \times m}$ is a positive definite diagonal matrix. To determine the state equations at the sliding motion, the equivalent control method is used. This consists of forcing of $\dot{S}\left(q_{e}, t\right)=0$, then determining the value of the equivalent control, and finally substituting it into the state equations. The derivative of $S\left(q_{e}, t\right)$ is

$$
\begin{aligned}
\dot{S}\left(q_{e}, t\right) & =H\left(q_{e}\right) \dot{q}_{e}-\dot{Z}_{s}\left(q_{e}, t\right) \\
= & H\left(q_{e}\right) g\left(q_{e}\right)\left(u_{\mathrm{disc}}\left(q_{e}, t\right)+g^{+}\left(q_{e}\right) P\left(q_{e}, t\right)\right) \\
& +H\left(q_{e}\right) P_{U}\left(q_{e}, t\right) .
\end{aligned}
$$

The equivalent control is defined as

$$
\begin{aligned}
u_{\mathrm{disc}}^{\mathrm{eq}}= & -\left(H\left(q_{e}\right) g\left(q_{e}\right)\right)^{-1} H\left(q_{e}\right) P_{U}\left(q_{e}, t\right) \\
& -g^{+}\left(q_{e}\right) P\left(q_{e}, t\right) .
\end{aligned}
$$

Substituting $u=u_{c}+u_{\text {disc }}^{\text {eq }}$ into system (1), we have

$$
\begin{aligned}
\dot{q}_{\mathrm{eq}} & \\
= & g\left(q_{e}\right) u_{c} \\
& +\left(I-g\left(q_{e}\right)\left(H\left(q_{e}\right) g\left(q_{e}\right)\right)^{-1} H\left(q_{e}\right)\right) P_{U}\left(q_{e}, t\right) .
\end{aligned}
$$

One has that the matched disturbance is eliminated, and the ISM control strategy has transformed the original uncertain term $P\left(q_{e}, t\right)$ into a new term:

$$
\begin{aligned}
P_{\mathrm{eq}} & \left(q_{e}, t\right) \\
& =\left(I-g\left(q_{e}\right)\left(H\left(q_{e}\right) g\left(q_{e}\right)\right)^{-1} H\left(q_{e}\right)\right) P_{U}\left(q_{e}, t\right) .
\end{aligned}
$$

An optimal choice of the state-dependent matrix $H\left(q_{e}\right)$ would minimize this term. The goal of the remainder of this work is then to solve the following problem.

Problem 4. For system (1) fulfilling Assumptions 1 and 3, find a function $S_{0}\left(q_{e}, t\right)$ such that

$$
\frac{d S^{*}\left(q_{e}, t\right)}{d q_{e}}=H^{*}\left(q_{e}\right)=\underset{H\left(q_{e}\right) \in \mathbb{R}^{m \times n}}{\arg \min }\left\|P_{\mathrm{eq}}\left(q_{e}, t\right)\right\|_{2} .
$$

2.2. The Proposed Sliding Manifold. A serval result is introduced for the minimization of the equivalent disturbance (18) for system (1), when the ISM control strategy is applied; hence, we consider the distribution given by

$$
\Lambda\left(q_{e}\right)=\operatorname{span}\left\{g^{\perp}\left(q_{e}\right)\right\} .
$$

That introduces the following assumption.

Assumption 5. $\Lambda\left(q_{e}\right)$ is involutive: that is,

$$
\left[g_{i}^{\perp}, g_{j}^{\perp}\right]=\frac{\partial g_{j}^{\perp}}{\partial q} g_{i}^{\perp}-\frac{\partial g_{i}^{\perp}}{\partial q} g_{j}^{\perp} \in \Lambda\left(q_{e}\right) \quad \forall i, j=1,2,
$$

where $[\cdot, \cdot]$ is the Lie bracket of two vector fields. Since Assumption 5 is fulfilled, there exists a function $\widetilde{S}_{0}\left(q_{e}, t\right)$ such that

$$
\frac{\partial \widetilde{S}_{0}\left(q_{e}, t\right)}{\partial q_{e}}=\widetilde{H}\left(q_{e}\right)=B\left(q_{e}\right) g^{T}\left(q_{e}\right)
$$




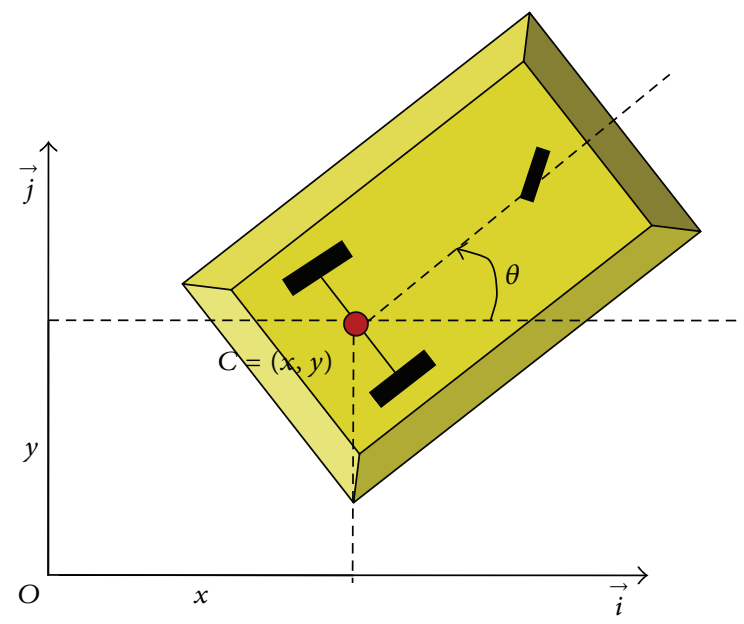

FIGURE 1: Kinematic model of unicycle-type mobile robot.

where $B(q) \in \mathrm{IR}^{m \times m}$ is a full rank matrix. Note that (22) guarantees that Assumption 3 holds.

The involutivity of $\Lambda\left(q_{e}\right)$ is equivalent to the existence of $m$ independent functions $\widetilde{S}_{0}\left(q_{e}, t\right)$ such that

$$
\frac{\partial \widetilde{S}_{0}\left(q_{e}, t\right)}{\partial q_{e}} g^{\perp}\left(q_{e}\right)=\widetilde{H}\left(q_{e}\right) g^{\perp}\left(q_{e}\right)=0
$$

Since $m$ columns of $\widetilde{H}^{T}\left(q_{e}\right)$ are independent, they span the orthogonal complement of $\Lambda\left(q_{e}\right)$. Recall that the double orthogonal complement of a closed subspace is equal to the subspace itself which is equivalent to

$$
\operatorname{span}\left\{\widetilde{H}^{T}\left(q_{e}\right)\right\}=\operatorname{span}\left\{g\left(q_{e}\right)\right\}
$$

The columns of $\widetilde{H}^{T}\left(q_{e}\right)$ and $g\left(q_{e}\right)$ are basis of the same subspace and the matrix $B^{T}(q)$ in (22) is simply the transformation matrix relating them.

\section{Kinematics Model of Wheeled Mobile Robot}

The kinematic model of unicycle-type wheeled mobile robot is described with consideration of the nonholonomic constraints. A complete study of the kinematics model of WMRs could be found in [16].

3.1. Kinematics Model of Unicycle-Type Wheeled Mobile Robot. A unicycle-type mobile robot is considered as depicted in Figure 1. The kinematic scheme of the robot consists of platform with two driving wheels mounted on the same axis with independent actuators and one free wheel (caster) is used to keep the robot stable [16]. It is assumed that the wheels are nondeformable and roll without lateral sliding.

The point $C$ at the center of the driving wheels axle is used as a reference point of the robot. The configuration of

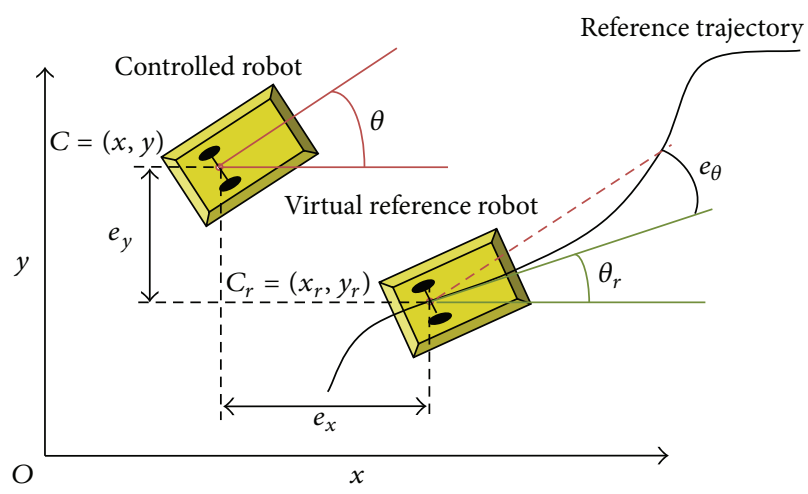

FIgURE 2: Tracking the reference trajectory of WMR.

the robot can be described by three generalized coordinates as

$$
q=(x, y, \theta)^{T} \in Q=\mathrm{IR}^{2} \times S O^{1},
$$

where $(x, y)$ are the coordinates of point $C$ and $\theta$ is the robot orientation. The nonholonomic constraint that the wheels cannot slip in the lateral direction is

$$
\left[\begin{array}{lll}
-\sin \theta & \cos \theta & 0
\end{array}\right]\left[\begin{array}{l}
\dot{x} \\
\dot{y} \\
\dot{\theta}
\end{array}\right]=0 .
$$

From this constraint, the kinematic model of the unicycle can be written as follows:

$$
\dot{q}=g(q) u \Longrightarrow\left[\begin{array}{l}
\dot{x} \\
\dot{y} \\
\dot{\theta}
\end{array}\right]=\left[\begin{array}{cc}
\cos \theta & 0 \\
\sin \theta & 0 \\
0 & 1
\end{array}\right]\left[\begin{array}{l}
v \\
w
\end{array}\right],
$$

where $v$ and $w$ area linear velocity of the wheels and its angular velocity, respectively. They are taken as control inputs $u=(v, w)^{T}$.

The driftless nonlinear system (27) has several control properties, most of which actually hold for the whole class of WRMs and nonholonomic mechanisms in general. This property comes from the fact that any position is an equilibrium point if the inputs are zero, and hence the system has no dynamical motion.

3.2. Posture Error Model of Mobile Robot. In trajectory tracking of wheeled mobile robots, the reference trajectory $q_{r}$ and the velocity $u_{r}$ are considered, where they are written as

$$
\begin{aligned}
q_{r} & =\left(x_{r}(t), y_{r}(t), \theta_{r}(t)\right)^{T}, \\
u & =\left(v_{r}(t), w_{r}(t)\right)^{T} .
\end{aligned}
$$

The mobile robot moves from posture $q$ to posture $q_{r}$, as shown in Figure 2. The posture error is given by

$$
\left(e_{x}, e_{y}, e_{\theta}\right)^{T}=\left(x-x_{r}(t), y-y_{r}(t), \theta-\theta_{r}(t)\right)^{T} .
$$




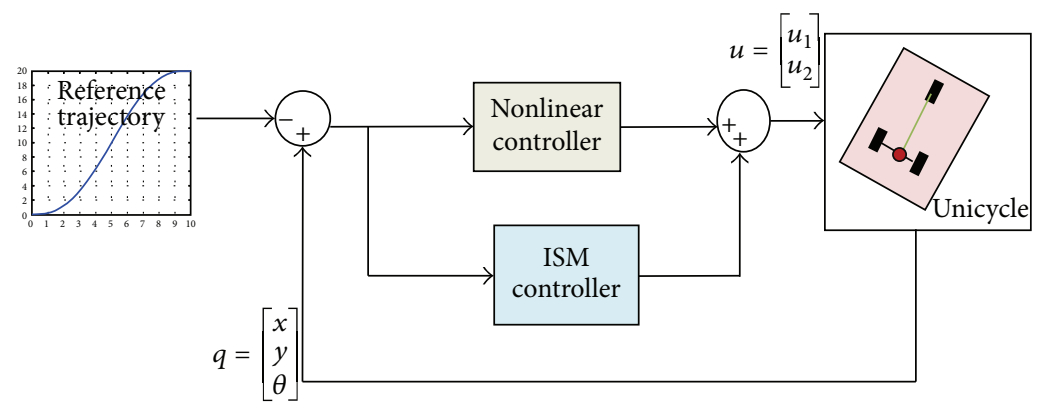

Figure 3: Closed-loop control diagram.

According to coordinate transformation, the posture error equation of the mobile robot is described as

$$
q_{e}=\left[\begin{array}{l}
x_{e} \\
y_{e} \\
\theta_{e}
\end{array}\right]=\left[\begin{array}{ccc}
\cos \theta_{r} & \sin \theta_{r} & 0 \\
-\sin \theta_{r} & \cos \theta_{r} & 0 \\
0 & 0 & 1
\end{array}\right]\left[\begin{array}{l}
e_{x} \\
e_{y} \\
e_{\theta}
\end{array}\right] .
$$

The derivative of the posture error given in (30) can be written as

$$
\dot{q}_{e}=\left[\begin{array}{c}
\dot{x}_{e} \\
\dot{y}_{e} \\
\dot{\theta}_{e}
\end{array}\right]=\left[\begin{array}{c}
y_{e} w_{r}-v_{r}+\cos \theta_{e} v \\
-x_{e} w_{r}+\sin \theta_{e} v \\
w-w_{r}
\end{array}\right] .
$$

For unicycle, it is assumed that $\left|\theta_{e}\right|<\pi / 2$, which means that the vehicle orientation must not be perpendicular to the desired trajectory. We can write (31) in the form affine in the control as follows:

$$
\begin{aligned}
\dot{q}_{e} & =f\left(q_{e}, t\right)+g\left(q_{e}\right) u \Longrightarrow \\
\dot{q}_{e} & =\left[\begin{array}{c}
\dot{x}_{e} \\
\dot{y}_{e} \\
\dot{\theta}_{e}
\end{array}\right]=\left[\begin{array}{c}
y_{e} w_{r}-v_{r} \\
-x_{e} w_{r} \\
-w_{r}
\end{array}\right]+\left[\begin{array}{cc}
\cos \theta_{e} & 0 \\
\sin \theta_{e} & 0 \\
0 & 1
\end{array}\right]\left[\begin{array}{l}
v \\
w
\end{array}\right] .
\end{aligned}
$$

The model of the unicycle usually has external disturbance or unmodeled uncertainty, so the behavior of (32) can be quite different from expected. If there is disturbances acting on the system, the dynamic of the posture error is given by

$$
\begin{aligned}
\dot{q}_{e}= & {\left[\begin{array}{c}
\dot{x}_{e} \\
\dot{y}_{e} \\
\dot{\theta}_{e}
\end{array}\right] } \\
= & {\left[\begin{array}{c}
y_{e} w_{r}-v_{r} \\
-x_{e} w_{r} \\
-w_{r}
\end{array}\right]+\left[\begin{array}{cc}
\cos \theta_{e} & 0 \\
\sin \theta_{e} & 0 \\
0 & 1
\end{array}\right]\left[\begin{array}{c}
v \\
w
\end{array}\right] } \\
& +\left[\begin{array}{c}
p_{1} \cos \theta_{e}-p_{2} \sin \theta_{e} \\
p_{1} \sin \theta_{e}+p_{2} \cos \theta_{e} \\
p_{3}
\end{array}\right] .
\end{aligned}
$$

From (3) and (4), we have

$$
\begin{aligned}
& g^{+}\left(q_{e}\right)=\left[\begin{array}{lll}
1 & 0 & 0 \\
0 & 0 & 1
\end{array}\right], \\
& g^{\perp}\left(q_{e}\right)=\left[\begin{array}{c}
-\sin \theta \\
\cos \theta \\
0
\end{array}\right], \\
& g^{\perp+}(q)=\left[\begin{array}{lll}
0 & 0 & 0 \\
0 & 1 & 0
\end{array}\right] .
\end{aligned}
$$

System (4) is written as

$$
\begin{aligned}
\dot{q}_{e}= & f\left(q_{e}, t\right)+g\left(q_{e}\right) u+P_{M}\left(q_{e}, t\right)+P_{u}\left(q_{e}, t\right) \\
& \Rightarrow \dot{q}_{e}=\left[\begin{array}{c}
\dot{x}_{e} \\
\dot{y}_{e} \\
\dot{\theta}_{e}
\end{array}\right] \\
= & {\left[\begin{array}{c}
y_{e} w_{r}-v_{r} \\
-x_{e} w_{r} \\
-w_{r}
\end{array}\right]+\left[\begin{array}{cc}
\cos \theta_{e} & 0 \\
\sin \theta_{e} & 0 \\
0 & 1
\end{array}\right]\left[\begin{array}{c}
v \\
w
\end{array}\right] } \\
& +\left[\begin{array}{c}
p_{1} \cos \theta_{e} \\
p_{1} \sin \theta_{e} \\
p_{3}
\end{array}\right]+\left[\begin{array}{c}
-p_{1} \sin \theta_{e} \\
p_{1} \cos \theta_{e} \\
0
\end{array}\right] .
\end{aligned}
$$

If we assume that each component of the vector $P$ is in absolute value smaller than a constant, $\bar{P}_{1}, \bar{P}_{2}, \bar{P}_{3}$, respectively, we obtain $D_{\text {sup }}=\sqrt{\bar{P}_{1}^{2}+\bar{P}_{2}^{2}+\bar{P}_{3}^{2}}$ as required in (2).

In the following, we applied the above control design (11) to this obtained model for the trajectory tracking.

\section{Integral Sliding Mode Controller Design Applied to the Unicycle}

In this section, the integral sliding mode controller (ISMC) is applied to a unicycle. Firstly, we mention the nonlinear timevarying continuous feedback based technique proposed by Nonami et al. [16], for using it as a nominal controller. Then, we determine the ISMC to assess the trajectory tracking in the presence of matched and unmatched perturbations from the initial condition (Figure 3). 
4.1. Nonlinear Time-Varying Feedback. Nonami et al. [16] proposed a nonlinear controller, which is globally stable around a reference trajectory, with the same nominal system structure described as in Figure 2. The Nonlinear State Tracking Control law developed in [16] can be given as

$$
\begin{aligned}
u_{c}\left(q_{e}, t\right) & =\left[\begin{array}{l}
v_{c} \\
w_{c}
\end{array}\right] \\
& =\left[\begin{array}{c}
v_{r}-k_{x}\left|v_{r}\right| x_{e} \\
w_{r}-k_{y} v_{r} \cdot y_{e}+k_{\theta}\left|v_{r}\right| \tan \left(\theta_{e}\right)
\end{array}\right],
\end{aligned}
$$

where $k_{x}, k_{y}$, and $k_{\theta}$ are positive constantthat can be calculated by eigenvalue placement and the first term in each velocity is a feedforward part.

This controller makes the origin of system (32) globally asymptotically stable if $v_{r}$ is a bounded differentiable function with bounded derivative and does not tend to zero when it tends to infinity. Readers are referred to [16] for detailed description. From (36) we note that $v_{r}$ can entirely pass through zero when it changes their sign.

4.2. Integral Sliding Mode Controller Design. The integral sliding mode control can be used to eliminate the effect of the matched disturbances and minimize the unmatched one. From the above design procedure, we define the control feedback as

$$
\begin{gathered}
v\left(q_{e}, t\right)=v_{c}\left(q_{e}, t\right)+v_{\mathrm{disc}}\left(q_{e}, t\right), \\
w\left(q_{e}, t\right)=w_{c}\left(q_{e}, t\right)+w_{\mathrm{disc}}\left(q_{e}, t\right) .
\end{gathered}
$$

To check if Assumption 5 is fulfilled, we consider the distribution:

$$
\Lambda\left(q_{e}\right)=\operatorname{span}\left\{g^{\perp}\right\}=\operatorname{span}\left\{\left[\begin{array}{c}
-\sin \theta \\
\cos \theta \\
0
\end{array}\right]\right\}
$$

The distribution $\Lambda\left(q_{e}\right)$ verifies Assumption 5. As a consequence, all the assumptions are fulfilled, and the minimization of the disturbance terms can be performed. Now we can calculate the sliding manifold by solving the equations in the form:

$$
\frac{\partial \widetilde{S}_{0}\left(q_{e}, t\right)}{\partial q_{e}} g^{T}\left(q_{e}\right)=0
$$

where $S_{0}=\left[\begin{array}{ll}S_{01} & S_{02}\end{array}\right]^{T}$ and from (39), we have

$$
\begin{aligned}
& {\left[\begin{array}{ccc}
\frac{\partial S_{01}}{\partial x} & \frac{\partial S_{01}}{\partial y} & \frac{\partial S_{01}}{\partial \theta} \\
\frac{\partial S_{02}}{\partial x} & \frac{\partial S_{02}}{\partial y} & \frac{\partial S_{02}}{\partial \theta}
\end{array}\right]\left[\begin{array}{c}
-\sin \theta \\
\cos \theta \\
0
\end{array}\right]=0} \\
& -\frac{\partial S_{01}}{\partial x} \sin \theta+\frac{\partial S_{01}}{\partial y} \cos \theta=0, \\
& -\frac{\partial S_{02}}{\partial x} \sin \theta+\frac{\partial S_{02}}{\partial y} \cos \theta=0 .
\end{aligned}
$$

Clearly, we can choose $S_{01}=\theta, S_{02}=x \cos \theta+y \sin \theta$ as an exact solution for (41) and (42). The sliding manifold is as follows:

$$
\widetilde{S}_{0}\left(q_{e}, t\right)=\left[\begin{array}{c}
\theta \\
x \cos \theta+y \sin \theta
\end{array}\right] .
$$

The partial derivative of $S_{0}\left(q_{e}, t\right)$ with respect to $q_{e}$ is

$$
\widetilde{H}\left(q_{e}\right)=\left[\begin{array}{ccc}
0 & 0 & 1 \\
\cos \theta & \sin \theta & -x \sin \theta+y \cos \theta
\end{array}\right] .
$$

From this choice of the integral sliding manifold, the system will reach the integral sliding manifold infinite time and remain on it in the presence of disturbances. On the other hand, from (9) we can see the zero initial value of $s\left(q_{e}, t\right)$ at $t=0$. Therefore, the sliding mode exists $\forall t \geq 0$.

\section{Simulation Results}

To assess the effectiveness of the proposed controller, computer simulations using MATLAB/SIMULINK are implemented. The simulations are performed by tracking a circular trajectory in which the desired position, orientation, and velocities of the unicycle are specified. This trajectory is described as

$$
\begin{aligned}
& x_{r}(t)=R \sin (\omega t), \\
& y_{r}(t)=R(1-\cos (\omega t)), \\
& \theta_{r}(t)=\omega t \\
& v_{r}(t)=R \omega \\
& w_{r}(t)=\omega .
\end{aligned}
$$




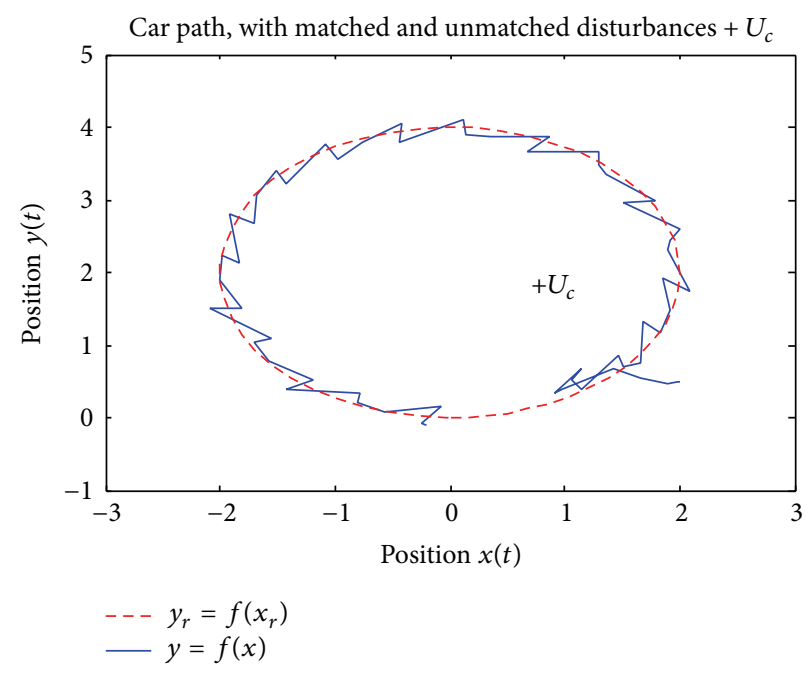

(a)

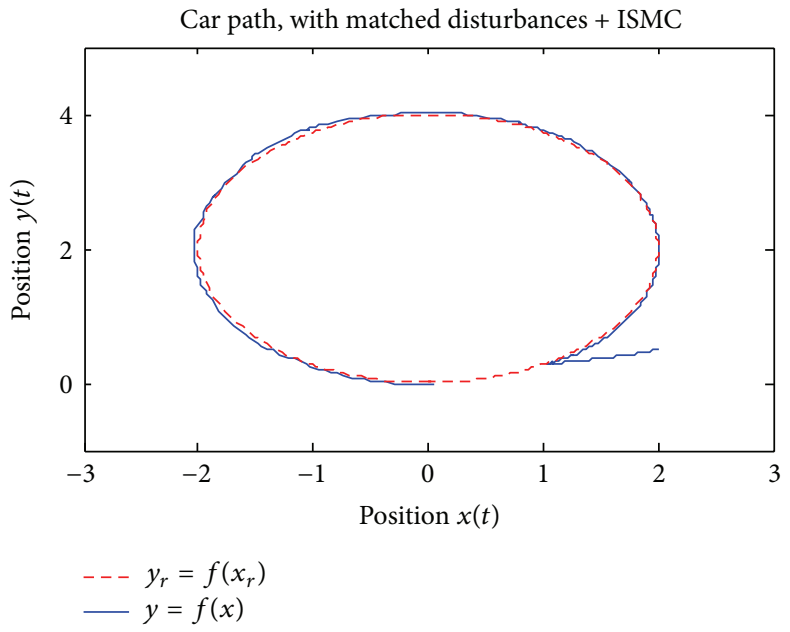

(b)

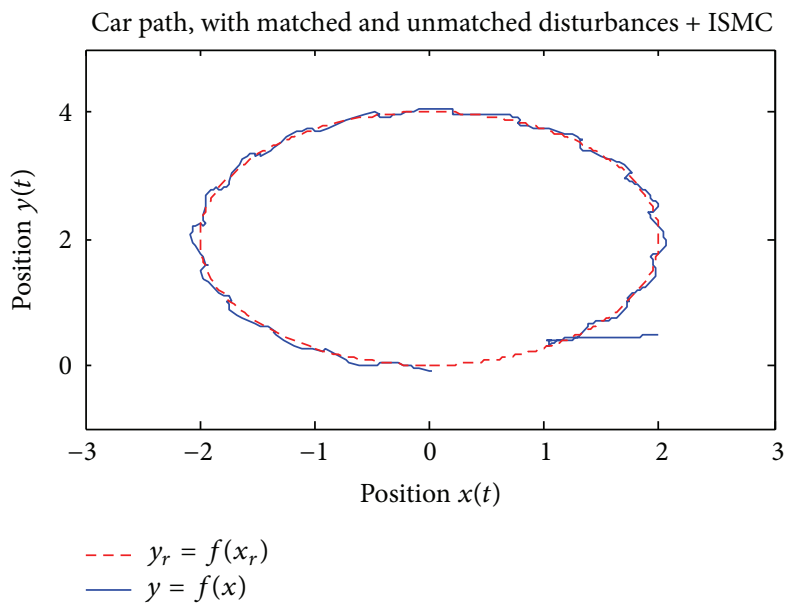

(c)

FIgURE 4: The path of the unicycle $x-y$ with initialization error and disturbances.

With initial configuration equal to $\left(x_{0}, y_{0}, \theta_{0}\right)=\left(0 \mathrm{~m}, 0 \mathrm{~m}, 0^{\circ}\right)$, and $R=2 \mathrm{~m}, \omega=1 \mathrm{rad} / \mathrm{s}$.

To show the robustness of our controller, the simulation was run with initialization error $\left(x_{0}, y_{0}, \theta_{0}\right)=$ $\left(2 \mathrm{~m}, 0.5 \mathrm{~m}, 20^{\circ}\right)$ and then with disturbances $P\left(q_{e}, t\right)$ such as

$$
P\left(q_{e}, t\right)=\left[\begin{array}{c}
2 \sin (20 t) \\
\sin (20 t) \\
0.8 \sin (8 t)
\end{array}\right] .
$$

Leading to $\Phi \approx 2.6$ in Assumption 1 , the gain is chosen by $D_{\text {sup }} \approx 2.6$. Moreover, in order to reduce the so-called chattering effect, the well-known equivalent control method is used, applying a linear low-pass filter to the obtained discontinuous control variable (Table 1).

First of all, we show (Figure 4(a)) the path of the unicycle in the $x-y$ plane in case there is matched and unmatched disturbance and the high-level controller only is used. As
TABLE 1: The parameter values of the control law used in the simulations.

\begin{tabular}{lccccc}
\hline Constants & $k_{x}$ & $k_{y}$ & $k_{\theta}$ & $M_{1}$ & $M_{2}$ \\
\hline$u_{c}\left(q_{e}, t\right)$ & 10 & 5 & 10 & - & - \\
$u_{\text {disc }}\left(q_{e}, t\right)$ & - & - & - & 3 & 3 \\
\hline
\end{tabular}

expected, after a transient (since the initial condition is taken on purpose different from the reference), the car trajectory (solid line) does not settle on the desired one (dashed line), and the high level controller has a poor performance, since it is not designed to work in presence of disturbances. Using the proposed ISM strategy, the bound on the matched disturbances is eliminated (Figures 4(b) and 5(a)) and the unmatched ones are not amplified (Figures 4(c) and 5(b)); the performance of the overall control law is improving. The unicycle with ISMC indicates a higher robustness, global stability, and higher tracking precision. 

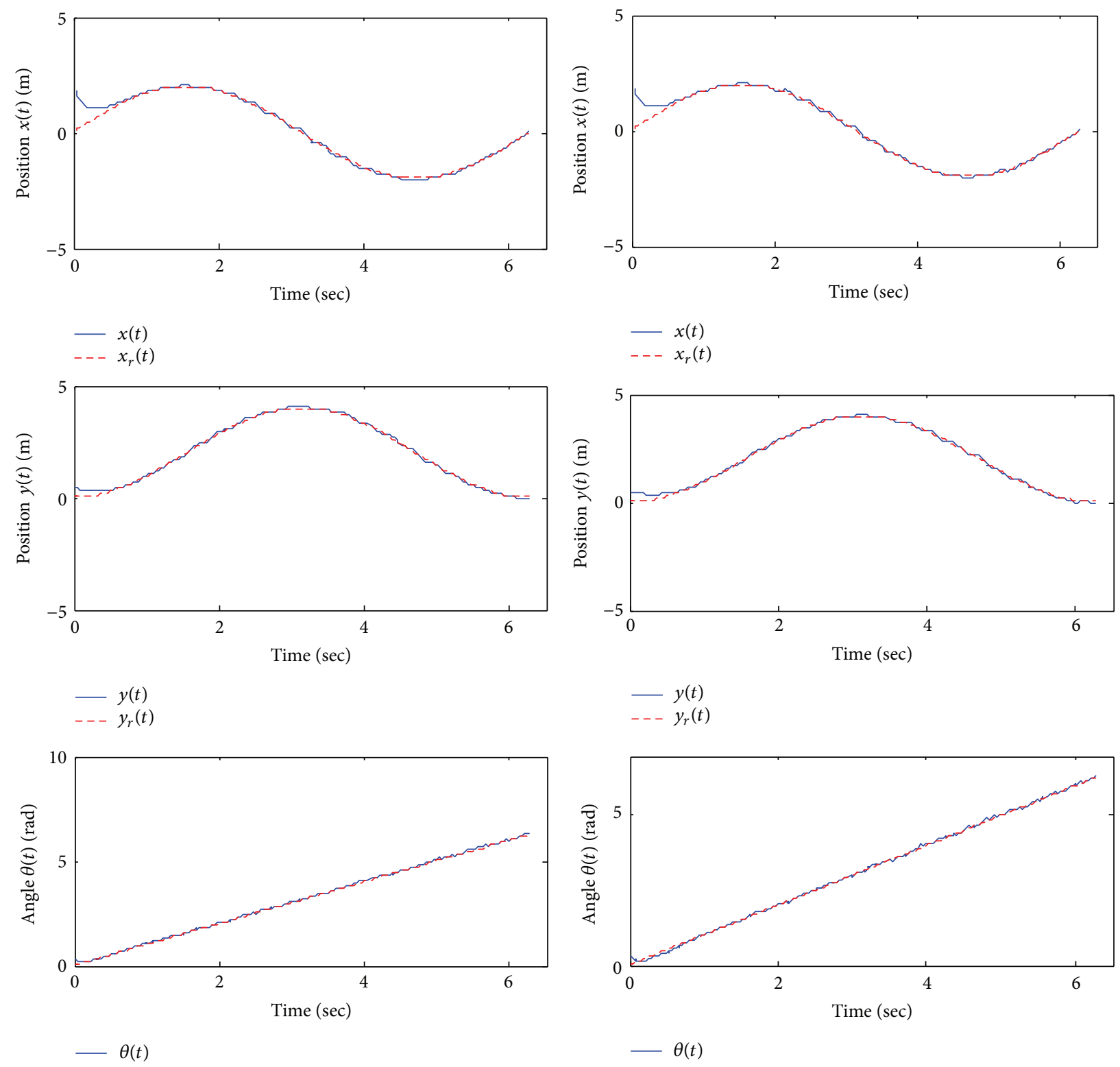

(a)

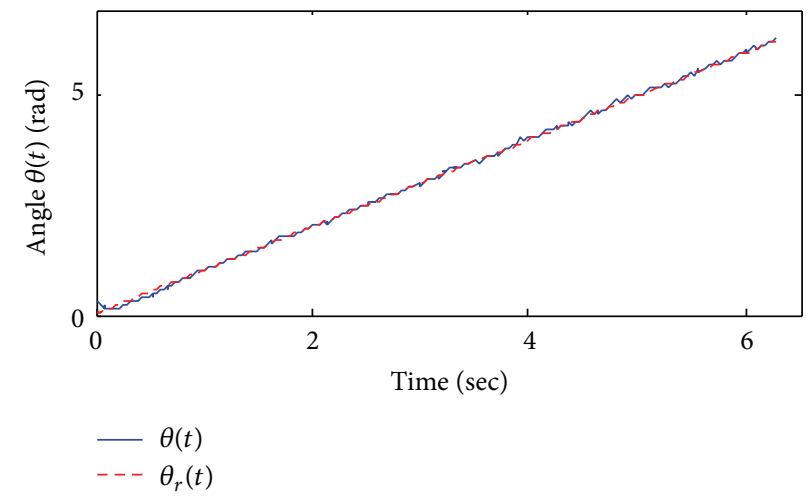

(b)

FiguRE 5: Tracking the reference trajectories $x(t), y(t)$, and $\theta(t)$, respectively, with initialization error. (a) In case of matched disturbances with ISMC and (b) in case of matched and unmatched disturbances with ISMC.

In this last case, we show also (Figure 7) the time evolution of the control variables $u_{1}$ and $u_{2}$. The time evolution of the components of the sliding manifold $s$ is steered to zero as shown in Figure 6, since the sliding mode is enforced from the initial instance.

\section{Conclusion}

In automatic control, the sliding mode control improves system performance by allowing the successful completion of a task even in the presence of perturbations. In this work, the uncertain system trajectories are asymptotically regulated to zero inspite, while a sliding mode is enforced in finite time along an integral manifold from the initial condition. The use of the integral sliding manifold allows one to subdivide the control design procedure into two steps. First high-level control and then a discontinuous control component are added so as to cope with the uncertainty presence. The design procedure is relying on the definition of a suitable sliding manifold and the generation of sliding modes as it guarantees the minimization of the effect of the disturbance terms, which takes place when the matched disturbances are completely rejected and the unmatched ones are not amplified. The performance of closed-loop system has been verified using 

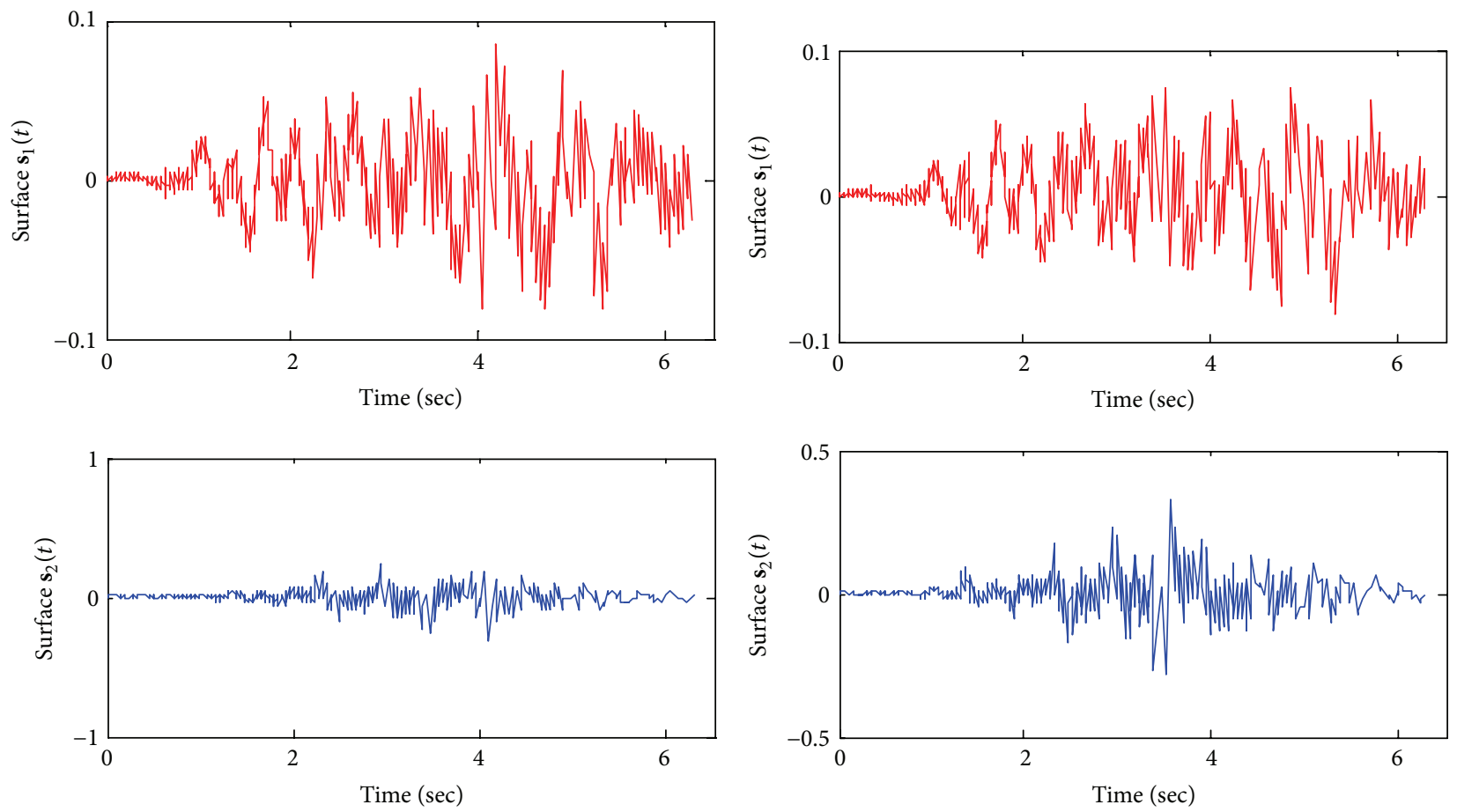

(a)

(b)

FIGURE 6: Time evolution of the components of sliding manifold $s_{1}$ and $s_{2}$, (a) in case of matched disturbances and (b) in case of matched and unmatched disturbances.
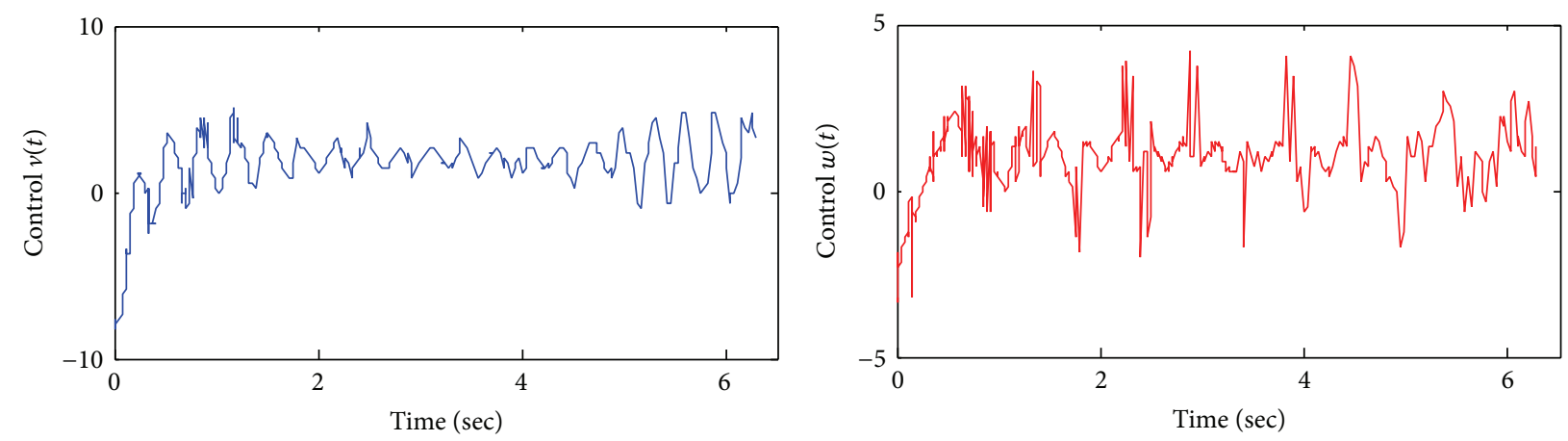

FIGURE 7: The control inputs $u_{1}$ and $u_{2}$ of the unicycle using integral sliding mode controller with matched and unmatched disturbances.

simulation results for the unicycle. In a future work, we will try to enhance this technique and to verify the obtained simulation results on the real test bench.

\section{Competing Interests}

The authors declare that there is no conflict of interests regarding the publication of this paper.

\section{References}

[1] K. Amar and S. Mohamed, "Stabilized feedback control of unicycle mobile robots," International Journal of Advanced Robotic Systems, vol. 10, article 187, 2013.
[2] A. Zdešar, I. Škrjanc, and G. Klančar, "Visual trajectorytracking model-based control for mobile robots," International Journal of Advanced Robotic Systems, vol. 10, article 323, 2013.

[3] R. Brockett, "Asymptotic stability and feedback stabilization," in Differential Geometric Control Theory, pp. 181-195, Birkhauser, Boston, Mass, USA, 1983.

[4] P. Morin and C. Samson, "Control of nonlinear chained systems: from the Routh-Hurwitz stability criterion to time-varying exponential stabilizers," IEEE Transactions on Automatic Control, vol. 45, no. 1, pp. 141-146, 2000.

[5] J. Ostrowski, "Steering for a class of dynamic nonholonomic systems," Transactions on Automatic Control, vol. 45, no. 8, pp. 1492-1498, 2000.

[6] E.-J. Hwang, H.-S. Kang, Ch.-H. Hyun, and M. Park, "Robust backstepping control based on a lyapunov redesign for 
skid-steered wheeled mobile robots," International Journal of Advanced Robotic Systems, vol. 10, article 26, 2013.

[7] F. Zouari, K. Ben Saad, and M. Benrejeb, "Robust adaptive control for a class of nonlinear systems using the backstepping method," International Journal of Advanced Robotic Systems, vol. 10, article 166, 2013.

[8] W.-J. Ever and H. Nijmeijer, "Practical stabilization of a mobile robot using saturated control," in Proceedings of the 45th IEEE Conference on Decision and Control (CDC '06), pp. 2394-2399, San Diego, Calif, USA, December 2006.

[9] V. Utkin, J. Guldner, and J. Shi, Sliding Modes in Electromechanical Systems, Taylor and Francis, London, UK, 2nd edition, 2009.

[10] J. Huang, Z.-H. Guan, T. Matsuno, T. Fukuda, and K. Sekiyama, "Sliding-mode velocity control of mobile-wheeled invertedpendulum systems," IEEE Transactions on Robotics, vol. 26, no. 4, pp. 750-758, 2010.

[11] W.-J. Cao and J.-X. Xu, "Nonlinear integral-type sliding surface for both matched and unmatched uncertain systems," IEEE Transactions on Automatic Control, vol. 49, no. 8, pp. 1355-1360, 2004.

[12] F. Castanos and L. Fridman, "Analysis and design of integral sliding manifolds for systems with unmatched perturbations," IEEE Transactions on Automatic Control, vol. 51, no. 5, pp. 853858, 2006.

[13] M. Defoort, T. Floquet, A. Kokosy, and W. Perruquetti, "Integral sliding mode control for trajectory tracking of a unicycle type mobile robot," Integrated Computer-Aided Engineering, vol. 13, no. 3, pp. 277-288, 2006.

[14] Ch.-Ch. Feng, "Integral sliding-based robust control," in Recent Advances in Robust Control, Novel Approaches and Design Methods, A. Mueller, Ed., pp. 978-953, InTech, 2011.

[15] M. Defoort, J. Palos, A. Kokosy, T. Floquet, and W. Perruquetti, "Performance-based reactive navigation for non-holonomic mobile robots," Robotica, vol. 27, no. 2, pp. 381-390, 2009.

[16] K. Nonami, M. Kartidjo, K. Yoon, and A. Budiyono, Autonomous Control Systems and Vehicle, Springer, Tokyo, Japan, 2013. 


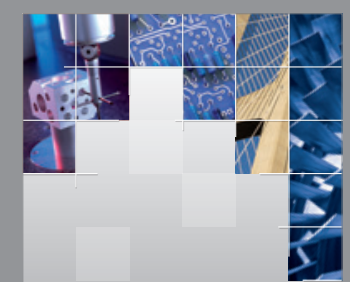

\section{Enfincering}
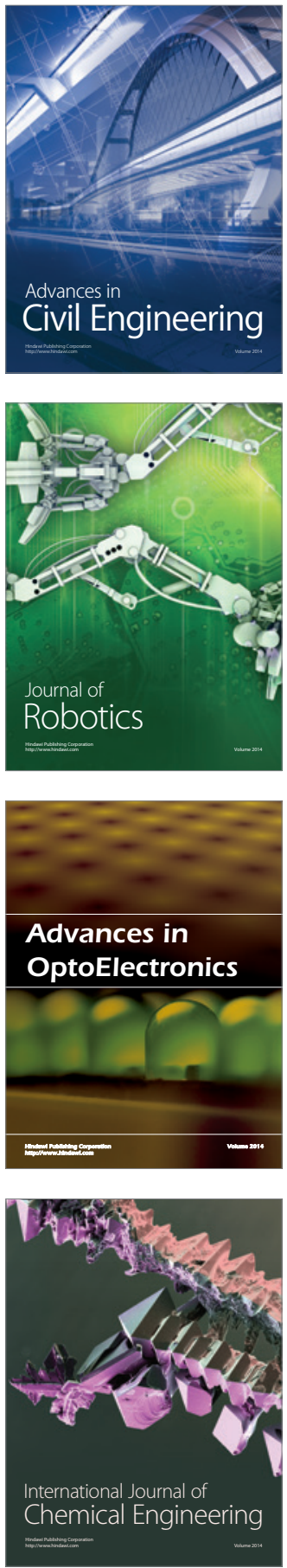

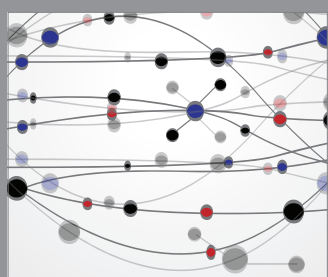

The Scientific World Journal

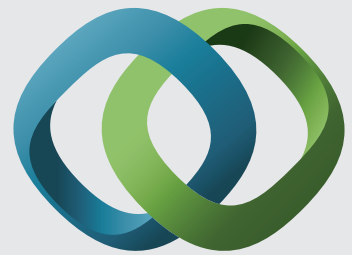

\section{Hindawi}

Submit your manuscripts at

http://www.hindawi.com
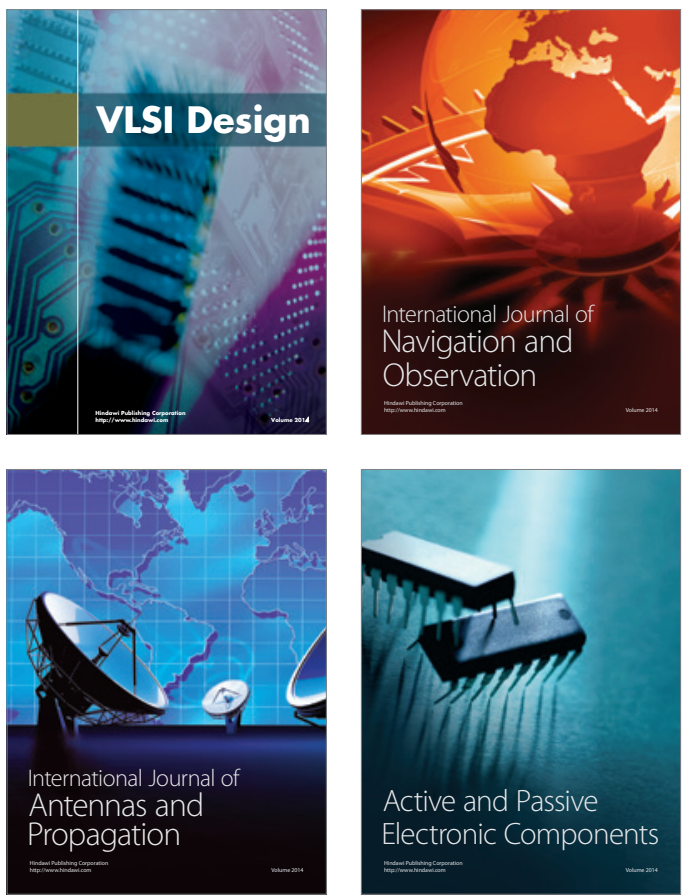
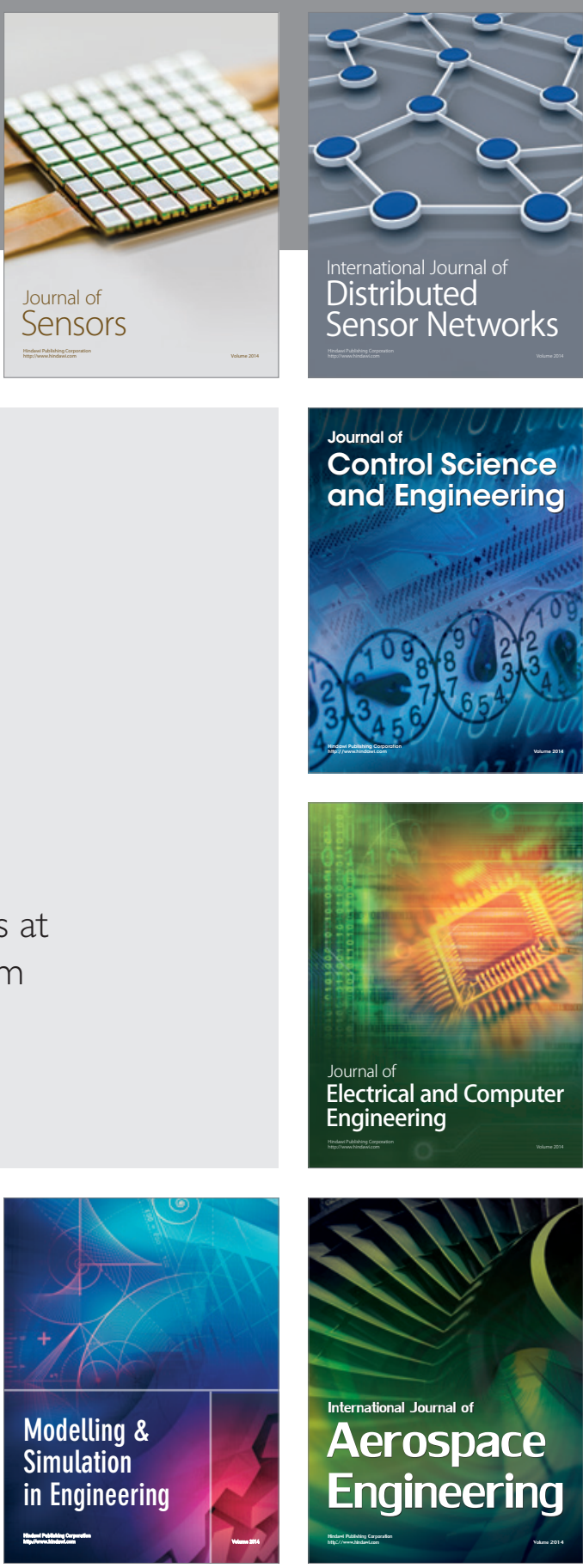

International Journal of

Distributed

Sensor Networks

Journal of

Control Science

and Engineering
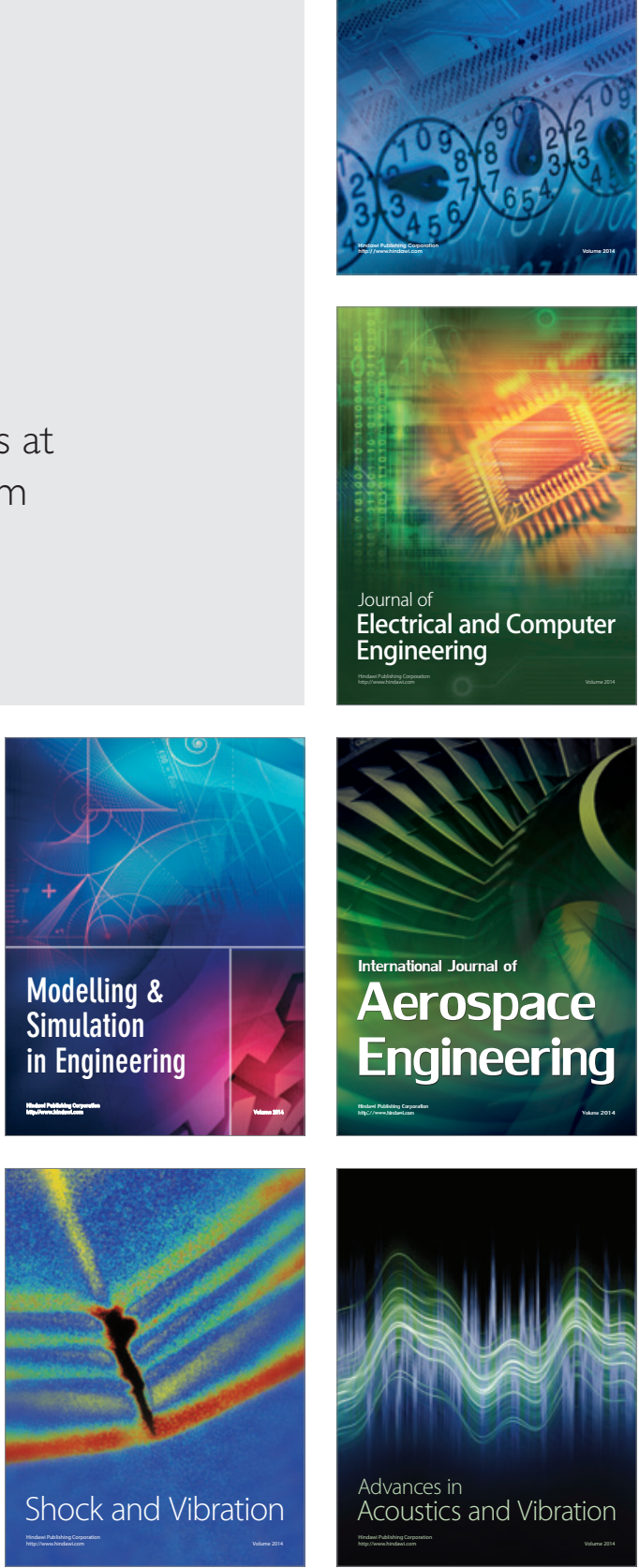